\title{
Fractional Order Modeling of PEMFC Temperature
}

\author{
Zhidong Qi, Dong Chen, Liang Shan \\ Automation Department \\ Nan Jing University of Science \& Technology \\ Nanjing, China \\ e-mail: qizhidong@sina.com, chendong_88@126.com,slshan@yahoo.com.cn
}

\begin{abstract}
The stack temperature of PEMFC is an important index of keeping the stability and efficiently of the fuel cell. Based on the conservation of energy and material balance equation, the theory of fractional calculus is firstly adopted to establish fractional order dynamic model of PEMFC temperature, and the method for solving the fractional differential equations is studied. The temperature variation is analyzed when the load current changes by simulation. The simulation results show that the fractional temperature model is feasible, and can reflect the stack temperature changing process accurately.
\end{abstract}

Keyword - PEMFC; Temperature; Modeling Fractional Order; Simulink

\section{I .INTRODUCTION}

PEMFC is a kind of fuel cell with low temperature, non-pollution, non-corrosive, high specific power, rapid-start, etc[1-2]. It has become one of the hot researches in the field of energy.Lots of research results show that, PEMFC stack temperature has a larger influence on the stack operation performance[3,4]. In order to further analyze the varying mechanism and the influence factors of the PEMFC temperature, it is necessary to establish an accurate model of the temperature.

The temperature dynamic model of PEMFC is described by an integer order calculus equations currently[4,5], but the real system are more or less affected by some non-integer order factors. Especially, the viscous substances with memory and hereditary, and the massive diffusion or thermal conductivity both belong to the typical fractional order systems or processes [6]. Described by integer order calculus equations, some real phenomena and properties of these systems or processes are often overlooked, and the actual system can't be described accurately by the integer model. Preliminary researches show that there exists a non-integer order relationship between the heat overflow and the stack temperature during the course of the thermal diffusion in fuel cell. Therefore, a fractional order dynamic model of PEMFC temperature based on the fractional calculus equations is proposed in this paper, and when the load current changes, the variation of the stack temperature is also analyzed by the simulation. The simulation results show that the fractional order model of PEMFC temperature can reflect the changing process of the stack temperature more accurately.

\section{II . Fractional Order Modeling of PEMFC TEMPERATURE}

\section{A. Theory of Fractional Order}

The fractional order calculus is a theory of arbitrary order differential and integral, and it is a generalization of the integer-order calculus. At present, the fractional order differential equation is used to establish a system of mathematical model in electrochemistry, thermal engineering, acoustic, electromagnetic, mechanical, control and other different fields in order to better describe the system dynamic characteristics.

The fractional order calculus is the theory that can solve any order of the derivative and integral, the continuous form of fractional calculus operator is defined as follows:

$$
{ }_{a} D_{t}^{\alpha}= \begin{cases}\frac{d^{\alpha}}{d t^{\alpha}}, & \operatorname{Re}(\alpha)>0 \\ 1, & \operatorname{Re}(\alpha)=0 \\ \int_{a}^{t}(d \tau)^{-\alpha}, & \operatorname{Re}(\alpha)<0\end{cases}
$$

Where a, $\mathrm{t}$ are the upper and lower limits of calculus; $\alpha$ is an arbitrary complex. In the development of the fractional order calculus theory, a lot of kinds of functions to define the fractional calculus are proposed, such as GL definition, RL definition, Caputo definition. The GL definition of fractional calculus is the most widely used, expressed as follows:

$$
{ }_{a} D_{t}^{\alpha} f(t)=\lim _{h \rightarrow 0} \frac{1}{h^{\alpha}} \sum_{j=0}^{[(t-a) / h]}(-1)^{j}\left(\begin{array}{l}
\alpha \\
j
\end{array}\right) f(t-j h)
$$

Where $w_{j}^{(\alpha)}=(-1)^{j}\left(\begin{array}{c}\alpha \\ j\end{array}\right)$ is the polynomial coefficients of the function $(1-z)^{\alpha}$, which can be calculated by the following recursive formula:

$$
w_{0}^{(\alpha)}=1, w_{j}^{(\alpha)}=\left(1-\frac{\alpha+1}{j}\right) w_{j-1}^{(\alpha)}, j=1,2, \ldots
$$

Eq.(2) can be rewritten as:

$$
{ }_{a} D_{t}^{\alpha} f(t)=\lim _{h \rightarrow 0} \frac{1}{h^{\alpha}} \sum_{j=0}^{[(t-a) / h]} w_{j}^{(\alpha)} f(t-j h)
$$

The formula (4) indicates that the fractional order differential value of the function at a certain moment is up to all of the previous function values of the sampling times, which shows that the fractional order differential is a local 
operator. It means that the fractional differential have a memory characteristic, which is obviously different from the integer order differential. Because of this feature, the fractional order differential has a unique advantage to establish a memory system model.

This paper adopts a method of the filter approximation to replace the fractional calculus operator. The performance of the filter Oustaloup is very outstanding among the many recursive filters [7].The corresponding filter can be written as:

$$
G_{f}(s)=K \prod_{k=-N}^{N} \frac{s+w_{k}}{s+w_{k}}
$$

Among them, the pole-zero and the gain can be calculated by the following formula:

$$
w_{k}=w_{b}\left(\frac{w_{h}}{w_{b}}\right)^{\frac{k+N+0.5^{*}(1-\gamma)}{2 N+1}}, w_{k}=w_{b}\left(\frac{w_{h}}{w_{b}}\right)^{\frac{k+N+0.5^{*}(1+\gamma)}{2 N+1}}, K=w_{h}^{\gamma}
$$

A filter can be constructed based on the above algorithm. When the signal $f(t)$ goes through the filter, the output of the filter can be approximated as $D_{t}^{\alpha} f(t)$. This paper will adopt this method to calculate the fractional calculus equations.

\section{B Fractional order modeling}

The stack temperature is an important parameter of the cell characteristics. When the stack temperature is low, the water is easy to concentrate; its static saturation pressure is small. At this time there will generate a flood phenomenon, all kinds of polarizations in the cell will enhance, the ohm impedance will increase, and the cell's performance will decline. The temperature increasing will reduce the ohmic resistance and the loss of polarization, which is good for the electrochemical reaction. But over high temperature will reduce the membrane water content, reduce the proton conductivity of membrane and increase the membrane resistance, this will leads to make the performance worse or even destroy the proton exchange membrane $[8,9]$. Therefore, it is very important to keep the PEMFC operating temperature at an ideal range.

According to the law of conservation of energy, the energy balance can be written as follows:

$$
\dot{Q}_{\text {absorb }}=Q_{\text {react }}-P_{\text {st }}-\dot{Q}_{\text {loss }}-\dot{Q}_{\text {cool }}
$$

Where $Q_{\text {react }}$ is the total power of the electrochemical

reaction; $P_{s t}$ represents the electrical load; $\dot{Q}_{\text {loss }}$ denotes the environmental thermal convection loss and radiation loss; $Q_{\text {cool }}$ is the heat loss of cooling system and $\dot{Q}_{a b s o r b}$ is the heat absorbed by the stack.

The temperature of the fuel cells and the heat absorbed by stack can be described with one- order differential equation:

$$
\dot{Q}_{a b s o r b}=M C_{t} \frac{d T}{d t}
$$

The paper [6] shows that there is a typically fractional relationship between the temperature and the heat diffusion in semi-solid. In the process of fuel cells running, there appears certain water content in the PEM, which presents a characteristic of semi solid. At this moment, there exists a fractional order property between the heat and temperature. So, in the process of thermal diffusion of the fuel cell, the non-integer order relationship between the heat and temperature can be described as follows[6]:

$$
\dot{Q}_{a b s o r b}=M C_{t} \frac{d^{n} T}{d t^{n}}
$$

According to Eq.(7),Eq.(9)can be further modified as:

$$
M C_{t} \frac{d^{n} T}{d t^{n}}=\dot{Q}_{\text {absorb }}=Q_{\text {react }}-P_{\text {st }}-\dot{Q}_{\text {loss }}-\dot{Q}_{\text {cool }}
$$

The total input power of the stack is directly related to the amount of hydrogen consumed; since the hydrogen consumption is dependent on the stack current and the number of cells, the following expression can be used to calculate the total power input:

$$
Q_{\text {react }}=N_{H_{2}, \text { used }} \times \Delta H=\frac{I \times N_{\text {cell }}}{2 F} \times \Delta H
$$

where $\Delta H$ is the combustion enthalpy of hydrogen, and $N_{H_{2} \text {,used }}$ is the flow rate of hydrogen.

The output electrical power is given by:

$$
P_{s t}=V_{\text {stack }} \times I
$$

Where I is the stack current, Vstack is the stack output voltage by the literature [10].

$$
V_{\text {stack }}=E_{\text {Nernst }}-V_{\text {act }}-V_{\text {ohm }}-V_{\text {con }}
$$

Where ENernst represents the Nernst voltage, which is also called irreversible open circuit voltage; Vact denotes the activation polarization; Vohm is the ohmic polarization and Vcon represents the concentration polarization.

The sum of environmental heat convection loss and radiation loss is expressed as:

$$
Q_{\text {loss }}=h A \cdot\left(T-T_{\text {room }}\right)+\sigma \cdot \varepsilon \cdot(T+273)^{4}
$$

Where $\mathrm{h}$ is the heat exchange coefficient, $\mathrm{A}$ is the surface area, Troom is the room temperature. Generally, compared with the other losses, the radiation loss of the fuel cell can be ignored.

Usually the heat losses of the cooling system can be expressed as:

$$
Q_{\text {cool }}=h A \times\left(T_{\text {air,out }}-T_{\text {air, }, \text { }}\right) \div\left(\ln \left(T-T_{\text {air }, \text { in }}\right)-\ln \left(T-T_{\text {air, out }}\right)\right)
$$

Tair,in and Tair,out are respectively the inlet temperature and outlet temperature of the cooling air.

\section{The Simulation of PEMFC TeMPERAture MODELING}

PEMFC is a nonlinear multi-input multi-output (MIMO) system. The input values include the moor flow rate and the pressure of the input gas, the temperature of the cool gas and the external load. The output values include the output voltage and the temperature of stack. By setting the 
simulation parameters, the temperature model of the integral order and the fractional order have been simulated and compared with the experimental data. The impact on the stack temperature by the load current and the order of the fractional order model also have been analyzed. In order to analyze the effectiveness and feasibility of the fractional order model of the temperature, three conditions are considered to study the temperature model of the integral order and fractional order.

A. When the input current is $5 A$, the changing curve of stack temperature is shown in Fig. 3.1 with different order ( $n=1,0.95,0.9$ in Eq.(10)).

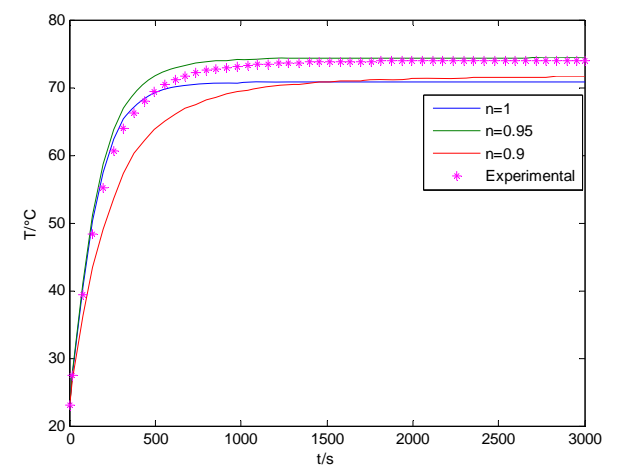

Fig.1 Curves of the stack temperature with different order

As shown in Fig. 1, the different curves can be obtained for different order. Compared with the integral order model, the fractional order model has a variable parameter $n$, which generates more changing curves. Thus, it can be seen that the fractional order model is closer to the actual object by choosing appropriate value of $n$.

$B$. When the load current is different, the curves of the stack temperature of the two models are shown in Fig. 2 and 3, respectively.

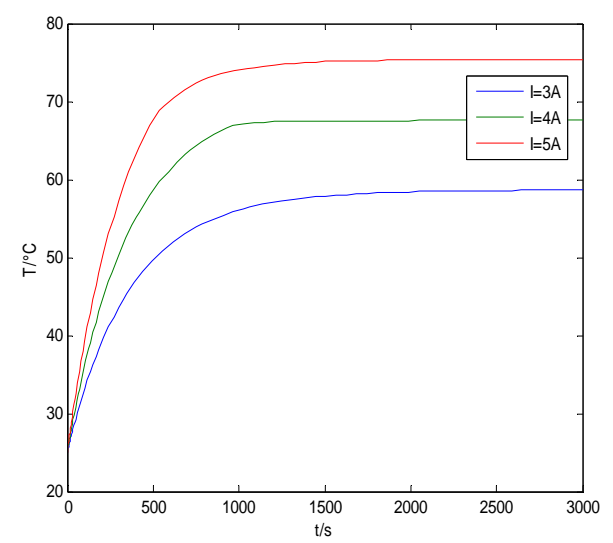

Fig.2 Temperature characteristic curves of fractional order model

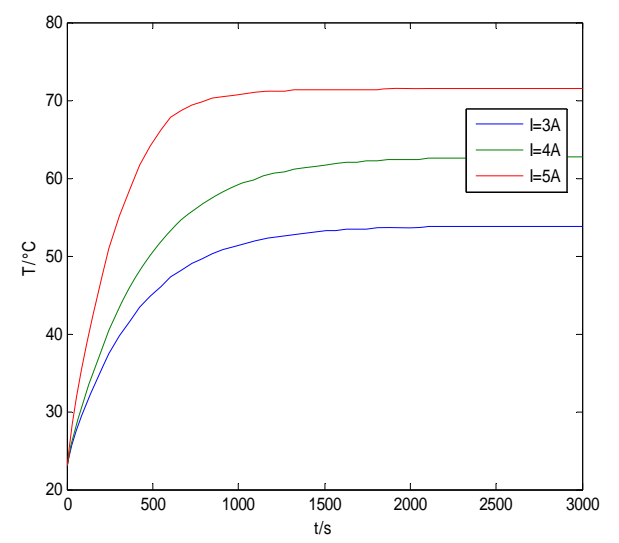

Fig.3 Temperature characteristic curves of integral order model

Fig.2 is the temperature changing curve of the fractional order model for different load currents when the parameter $n=0.9$. And Fig. 3 is the temperature changing curve of the integral order model for different load currents. The rapidity of system is improved and the stable temperature rises in both of the two models with the increasing of the load current. The difference is that the curves rise more rapidly and reach the stable value more quickly in the fractional order model.

C. When the load current continuously change, the temperature changing curve of the integral order model and fractional order model are studied. The step input signal of the load current Fig. 4 is used to simulate. The stack current firstly rises from $3 \mathrm{~A}$ to $5 \mathrm{~A}$ at 1000 s and then reduces to $1 \mathrm{~A}$ at 2000s. The simulation result is shown in Fig.5.

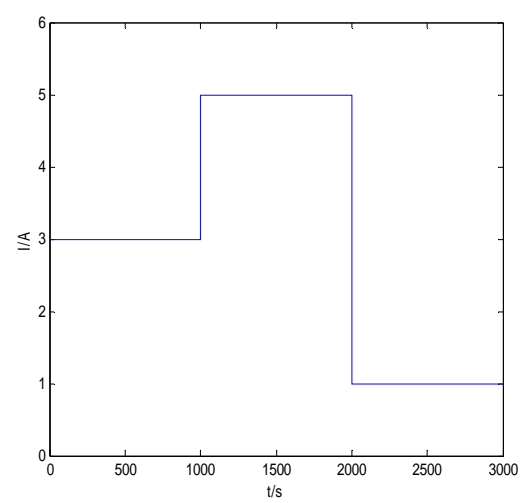

Fig. 4 Step input signal of the load current 


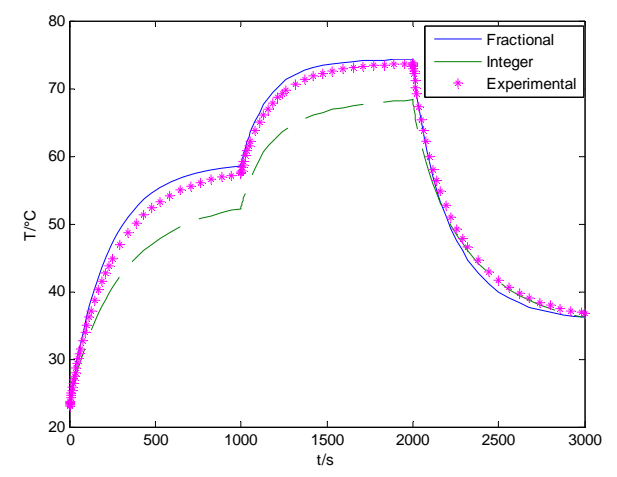

Fig.5 The response of stack temperature on difference load

Fig.5 shows the temperature changing curve of the integral order model and the fractional order model with changing load. The stack current firstly rises from 3A to 5A at $1000 \mathrm{~s}$. The temperature of the fractional order model rise from $60^{\circ} \mathrm{C}$ to $75^{\circ} \mathrm{C}$ and is gradually stabilized. Meanwhile, the temperature of the integral order model rises from $53^{\circ} \mathrm{C}$ to $68^{\circ} \mathrm{C}$ and also is gradually stabilized. When the load current decreases from $5 \mathrm{~A}$ to $1 \mathrm{~A}$ at 2000 s, the temperature of the two models both decreases to $36^{\circ} \mathrm{C}$ and is gradually stabilized.

It can be seen clearly that the curve of fractional order model is more accurate by comparing with the experimental data. Under the same circumstances, the fractional order model which has one more variable parameter $\mathrm{n}$ is closer to the actual data comparing with the integral order model by choosing a proper value of $n$. The value of $n$ can be selected by parameters identification, but makes the fractional order model more complex than the integral order model.

\section{CONCLUSION}

According to the heat transfer mechanism of PEMFC, a fractional order mechanism model of the temperature is firstly established based on the theory of the fractional calculus, which is compared with the integer order model. And then the solution method of the fractional order calculus is studied in this paper, the result of the simulation shows that the curve of the fractional model is more abundant, more realistic than the integer order model. Especially while the load current changes, the temperature curve of the fractional order model can quickly responds the change of the load current, and the changing process of the stack temperature is more accurately. The fractional model proposed in this paper provides a good foundation for the design of the advanced control algorithms of the stack temperature.

\section{ACKNOWLEDGMENT}

The project is supported by National Natural Science Foundation of China (NO.61203009, 61374153), China Postdoctoral Science Foundation (NO. 2012M511763), and Jiangsu Postdoctoral Science Foundation (NO. 1101085C).

\section{REFERENCES}

[1] Xu Lamei. Simulation and Optimization Of Proton Exchange Membrane Fuel Cell , Beijing: Beijing: national defence industry press, 2012.8.,pp.

[2] Zhang Liyan, Quan Shuhai. Modeling Of The Fuel Cell System Modeling And Optimization Control , Beijing: electronic industry press, 2011.11.pp.

[3] Hu Peng, Cao Guangyi, Zhu Xinjian, "Proton Exchange Membrane Fuel Cell Temperature Model and The Fuzzy Control. Control theory and application ,”. vol. 28, no. 10, 2011.

[4] Chen Weirong,Zhang Hanyue,Li Qi, “The Analysis Of Comprehensive Dynamic Model Of Proton Exchange Membrane Fuel Cell.Journal of southwest jiaotong university,” . vol. 47, no. 6, 2012.

[5] J. Kim, M. Lees, S. Srinivasan. "Modeling of Proton Exchange Membrane Fuel Cell Performance with an Empirical Equation” . Electrochem Soc, 1995, 142: 670-2674.

[6] Chen Yang-Quan, Kevin L.Moore, “Analytical stability bound for a class of delayed fractional-order dynamic systems”.Proc.of the 40th IEEE Conference, Decision and Control'01, vol.2, pp.1421-1426, 2001

[7] Mann R F, Amphlettj J C, Hooper M A I, et al. "Development and application of a generalized steady-state electrochemical model for a PEM fuel cell,”. Journal of Power Sources, 2000, 86(1/2): 173-180.

[8] Amphlett J C, Baumert R M, Mann R F, et al. "Performance modeling of the Ballard mark-IV solid polymer electrolyte fuel cell,” Journal of Electrolchemical Society, 1995, 142(1): 9-15.

[9] Dutta S, Shimaplee S, Van Zee J. "Numerical prediction of mass-exchange between cathode and anode channels in a PEM fuel cell ,’. International Journal of Heat and Mass Transfer, 2001, (44):2029-2042.

[10] Murgia G, Pisani L, Valentini M, et al "Electrochemistry and mass transport in polymer electrolyte membrane fuel cells," Journal of Electrochemical Society, 2002, 149(1): A31-A381. 